Jan Ruckenbiel

Gesundheit, Arbeit und Zusammenhalt 
Soziologische Studien

Band 45 
Jan Ruckenbiel

\title{
Gesundheit, Arbeit und Zusammenhalt
}

\author{
Betriebliche Gesundheitsförderung \\ durch Arbeitsgestaltung
}

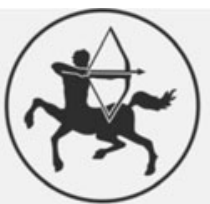

Centaurus Verlag \& Media UG 


\section{Zum Autor}

Jan Ruckenbiel wurde in Norddeutschland geboren. Geprägt durch intensive Arbeitserfahrungen im Hamburger Hafen interessierte er sich schon früh für arbeitsmedizinische Fragen. Nach dem Studium arbeitete er zuerst in verschiedenen Projekten der Empirischen Sozialforschung und wissenschaftlichen Begleitung, oft an der Nahtstelle von Wirtschaft und Gesellschaft und mit quantifizierenden Methoden. Er lebt heute im Rheinland und ist als promovierter Sozialwissenschaftler im Betrieblichen Gesundheitsmanagement tätig.

\section{Bibliografische Informationen der Deutschen Nationalbibliothek} Die Deutsche Nationalbibliothek verzeichnet diese Publikation in der Deutschen Nationalbibliografie; detaillierte bibliografische Daten sind im Internet über http://dnb.d-nb.de abrufbar.

ISBN 978-3-86226-231-1

ISBN 978-3-86226-986-0 (eBook)

DOI 10.1007/978-3-86226-986-0

ISSN 0937-664X

Gedruckt auf säurefreiem und chlorfrei gebleichtem Papier.

Alle Rechte, insbesondere das Recht der Vervielfältigung und Verbreitung sowie der Übersetzung, vorbehalten. Kein Teil des Werkes darf in irgendeiner Form (durch Fotokopie, Mikrofilm oder ein anderes Verfahren) ohne schriftliche Genehmigung des Verlages reproduziert oder unter Verwendung elektronischer Systeme verarbeitet, vervielfältigt oder verbreitet werden.

(C) CENTAURUS Verlag \& Media KG, Freiburg 2013

www.centaurus-verlag.de

Satz: Vorlage des Autors

Umschlaggestaltung: Jasmin Morgenthaler, Visuelle Kommunikation

Umschlagabbildung: OGOWIN - Stahlgießerei Friedrich-Wilhelms-Hütte Mühlheim. Mitarbeiter beim Abguss der Formen auf der Formanlage. Bild-ID 642. Copyright BMU/Oberhäuser 
Mein Dank gilt allen, die durch ihr Mitwirken und ihr Dasein zum Werden des Werkes beigetragen haben.

Möge es als Baustein für den weiteren Ausbau gesunden Arbeitens dienen. 
Diese Seite wurde absichtlich freigelassen 


\section{Inhaltsverzeichnis}

I. EINLEITUNG 1

II. MENSCH UND GESUNDHEIT 9

1. Zum Begriff der Gesundheit 9

2. Salutogenese $\quad 11$

2.1 Generalisierte Widerstandsressourcen und soziales Kapital 13

2.2 Der Kohärenzsinn: Verstehbarkeit, Handhabbarkeit, Sinnhaftigkeit 20

2.3 Stressorbewertung 26

2.4 Ergänzende Stresskonzepte 31

2.5. Zur Gesundheitswirksamkeit von Vertrauen, sozialer Unterstützung und $\begin{array}{ll}\text { Gruppenkohäsion } & 38\end{array}$

2.6 Resilienz und Fehlerfreundlichkeit $\quad 52$

2.7 Arbeitsethik und Arbeitssucht 54

3. Das Anforderungs-Kontroll-Modell 63

4. Das Modell beruflicher Gratifikationskrisen $\quad 65$

5. Empirische Evidenz und Anmerkungen zu den Modellen 68

III. FORSCHUNGSSTAND UND METHODIK DER EMPIRISCHEN $\begin{array}{ll}\text { UNTERSUCHUNG } & 77\end{array}$

1. Handlungsspielraum und Gruppenkohäsion als Moderatoren der $\begin{array}{ll}\text { Arbeitsüberlastung } & 77\end{array}$

2. Das Untersuchungsmodell: Demand-Control-Cohesion 79

3. Datenerhebung, Konstruktion der Maße und statistisches Vorgehen $\quad 85$

4. Hypothesen $\quad 92$ 


\section{HANDLUNGSSPIELRAUM UND GRUPPENKOHÄSION ALS}

MODERATOREN DER ARBEITSÜBERLASTUNG

$\begin{array}{ll}\text { 1. Ergebnisse } & 93\end{array}$

$\begin{array}{lr}\text { 2. Diskussion } & 103\end{array}$

$\begin{array}{lr}\text { 3. Zusammenfassung } & 121\end{array}$

V. ORGANISATION, UMWELT UND GESUNDHEIT ARBEITSBEZOGENE WECHSELWIRKUNGEN ZWISCHEN MENSCH,WIRTSCHAFT UND GESELLSCHAFT

1. Organisation

1.1 Zur Koordination intraorganisationalen Handelns: Rollen, Arbeitsanforderungen und Zielvereinbarungen

1.2 Kommunikation: Individualisierungstendenzen der Arbeitsbelastung 135

1.3 Handlungsspielräume: Gesundheitsbrunnen und Unsicherheitsquelle 138

1.4 Zur Soziogenese von Emotionen, Stress und Burnout am Arbeitsplatz 140

2. Betriebliches Gesundheitsmanagement

3. Gesellschaft, Arbeit, Individualisierung und Kohäsion

3.1 Die Gesellschaft als Bühne gesunden Lebens 151

3.2 Die neue Unsicherheit der Arbeit und der Arbeitenden 154

3.3 Ungenügenheitserleben und generalisierte Lebensunsicherheit. Risiken von Globalisierung und Beschleunigung

3.4 Gesundheit als kollektive Herausforderung 159

4. Die gesunde Organisation - zwischen Mensch und Umwelt 159

4.1 Reboundeffekte zwischen Organisation und Umwelt 159

4.2 Ziele und Zielkonflikte: Profit, Legitimität und Gesundheit $\quad 160$

4.3 Die reziproke Organisation: Sinn erschließen, Verstehbarkeit fördern, Handhabbarkeit sichern 
VIII. LITERATURVERZEICHNIS

IX. VORABVERÖFFENTLICHUNGEN

212

X. ANHANG

213

XI. ABKÜRZUNGSVERZEICHNIS

220 
Diese Seite wurde absichtlich freigelassen 


\section{Verzeichnis der Abbildungen, Graphiken und Tabellen}

\section{Abbildungen}

Abb. 2.1: Klassifikation von Stressbewältigungsstrategien

Abb. 2.2: Arten der Stressbewältigung

Abb. 2.3: Einwirkungen Dritter auf Stresswahrnehmung und

Stressentstehung

Abb. 3.1: Darstellung der Moderatorbeziehung in Modellen

Abb. 3.2: Der Einfluss von Arbeitsüberlastung, Entscheidungsspielraum und Gruppenkohäsion auf die Gesundheit

Abb. 5.1: Modell der organisationalen Bewältigung

Abb.10.1: Gesundheitspotentiale und Gesundheitsressourcen bei der Stressbewältigung

Abb.10.2: Einwirkungen Dritter auf Stresswahrnehmung und

Stressentstehung (ausführlichere Darstellung)

\section{Graphiken}

Graphik 1: Arbeitsüberlastung

Graphik 2: Gruppenkohäsion

96

Graphik 3: Entscheidungs- und Kontrollspielraum

Graphik 4: Zerssens Beschwerdenliste

\section{Tabellen}

Tabelle 1: Kennwerte der eingesetzten Items und Skalen

Tabellen 2a bis Tabelle 5: Lineare Regressionen auf Zerssens Symptome Tabelle 2a: Handlungsspielraum, Arbeitsüberlastung und Gesundheit 
Tabelle 2d: Kollinearitätsdiagnose

Tabelle 3a: Handlungsspielraum als Moderator $\quad 100$

Tabelle 3b: Gruppenkohäsion als Moderator $\quad 100$

Tabelle 4: Gruppenkohäsion \& Handlungsspielraum als Moderatoren 101

Tabelle 5: Handlungsspielraum, Gruppenkohäsion \& Arbeitsüberlastung 102 